\title{
Optimizing the parameters of Sugeno based adaptive neuro fuzzy using artificial bee colony: A Case study on predicting the wind speed
}

\author{
Fatma Helmy Ismail *, Mohamed Abdel Aziz ${ }^{\dagger}, \S$ Aboul Ella Hassanien ${ }^{\ddagger}$, \\ * Faculty of Computer Science Misr International University, Egypt \\ $\dagger$ Faculty of Sceince, Zagazig University, Egypt \\ \# Faculty of Computers and Information, Cairo University, Egypt \\ $\S$ Faculty of Computer science, Nahda University, Beni Suef, Egypt.
}

\begin{abstract}
This paper presents an approach based on Artificial Bee Colony $(\mathrm{ABC})$ to optimize the parameters of membership functions of Sugeno based Adaptive Neuro-Fuzzy Inference System (ANFIS). The optimization is achieved by Artificial Bee Colony (ABC) for the sake of achieving minimum Root Mean Square Error of ANFIS structure. The proposed ANFIS-ABC model is used to build a system for predicting the wind speed. To ensure the accuracy of the model, a different number of membership functions has been used. The experimental results indicates that the best accuracy achieved is $98 \%$ with ten membership functions and least value of RMSE which is 0.39 .

Index Terms-Wind Speed Prediction, Adaptive Network Based Fuzzy Inference System (ANFIS), Artificial Bee Colony (ABC), Swarm Intelligence, Root Mean Square Error (RMSE).
\end{abstract}

\section{INTRODUCTION}

A DAPTIVE Neuro-Fuzzy Inference Systems (ANFIS)[1] can be used for energy planning. Its learning techniques is to adjust the parameters of FIS membership functions that best represent the given input/output data. An adaptive neuro fuzzy control [2] is applied to optimize the use of wind energy in smart grids. In [3] the ANFIS have have applied for wind power prediction. Also, the authors in [4] predicted the wind speed using soft computing models formulated on a back propagation neural network (BPNN) and an adaptive neurofuzzy inference system (ANFIS). The adaptive neurofuzzy inference system (ANFIS) has been applied to estimate optimal power coefficient value of the wind turbines by [5]. In [6] the fuzzy modeling techniques and artificial neural networks have applied to estimate annual energy output of a wind turbine. the authors in [7] have demonstrated an online fuzzy neural network controller for output maximization in a wind energy conversion system. An on-line training recurrent fuzzy neural network (RFNN) controller for wind generation system with a high-performance model reference adaptive system (MRAS) observer for the sensorless control of an induction generator (IG) have presented [8]. In [9,10] ANFIS have applied for wind speed profiling and for wind power prediction. In [11] the wind speed is predicted using fuzzy logic and artificial neural network.

The artificial bee colony (ABC) algorithm is relatively a new swarm intelligence based optimizer [12][13]. Some good properties of $\mathrm{ABC}$ has been revealed in[14][16]. Especially, the number of controlling parameters in $\mathrm{ABC}$ is less than that of other population-based algorithms, which makes it easier to be implemented. Moreover, the optimization performance of $\mathrm{ABC}$ is comparable and sometimes superior to the state-of-theart meta-heuristics. That is why $\mathrm{ABC}$ has aroused interest and has been successfully applied to different kinds of optimization problems [17][19].

This paper presents an application of ANFIS-ABC to predict the wind speed. Where the ABC algorithm is applied to search the optimal parameters of ANFIS structure. The best parameters of membership functions are adjusted again using ABC.

Section II presents an outline of ANFIS structure. Section III introduces the proposed ANFIS-ABC structure. Section IV demonstrates the experimental results. Section V shows the implementation and results of building a wind speed prediction system. Section VI introduces future work and conclusion.

\section{ANFIS}

ANFIS is an adaptive fuzzy inference system [1]. The architecture of ANFIS is shown in Fig (1). The first two stages of the fuzzy inference process are fuzzifying the inputs and applying the fuzzy operator. The output of Sugeno membership functions are either linear or constant. The rule in a Sugeno fuzzy model has two main components, the antecedent and the consequent parts and has the form

If $x_{1}$ is $A_{i 1}$ and $x_{2}$ is $A_{i 2}$, then $y_{i}$ is $f_{i}(\mathrm{x})$

where $x_{1}, x_{2}$ are the input variables to the ANFIS. $A_{i 1}, \ldots, A_{\text {in }}$ are the linguistic variables of input membership function for the ith rule $(i=1,2, \ldots, n)$ and $y_{i}$ is the consequent part of $i$ th rule. The fuzzy set $A_{i j}$ at layer one uses a Gaussian membership function for each input variable and it has the form shown in $\mathrm{Eq}(1)$

$$
A_{i j}(x)=e^{-\left(\frac{x_{j}-m_{i j}}{\sigma_{i j}}\right)^{2}}
$$

where $m_{i j}$ and $\sigma_{i j}$ are the center and the width of the fuzzy set $A_{i j}$ respectively. The parameters of this layer are the antecedent parameters. The output of the fuzzy inference system with $n$ rules is calculated by weighting the real 
values of consequent parts of all rules with the corresponding membership grade is shown in $\operatorname{Eq}(2,3,4)$

$$
\hat{y}=\sum_{i=1}^{n}\left(\bar{w}_{i} f_{i}\right)=\frac{w_{i}}{\sum_{i=1}^{n}\left(w_{i}\right)}
$$

where

$$
w_{i}=\prod_{j=1}^{n} A_{i j}\left(x_{i}\right)
$$

and

$$
y_{i}=f_{i}(x)=a_{i} x_{1}+b_{i} x_{2}+c_{i}
$$

where $a_{i}, b_{i}$ and $c_{i}$ are the set of consequent parameters.

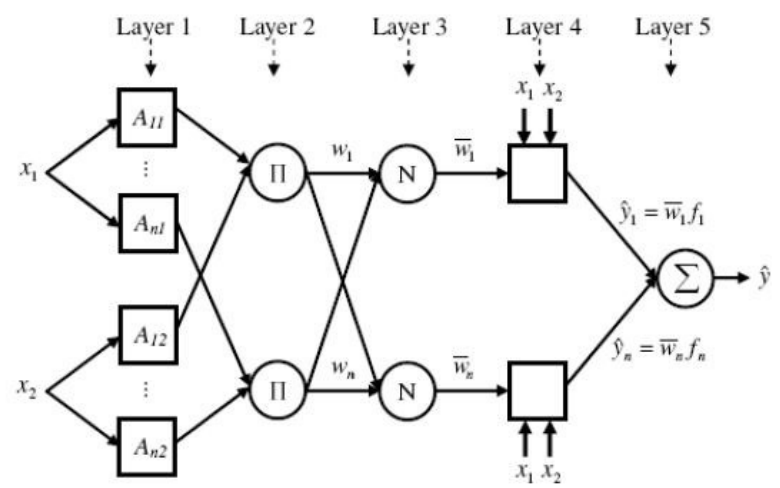

Fig. 1: Sugeno-Type Fuzzy Inference

\section{PRoposed ANFIS-ABC}

In Artificial Bee Colony (ABC), the colony contains three groups: employed bees, onlookers and scouts. The first half of the colony consists of employed bees and the second half contains onlookers. Employed bees search for food sources and share the information about these sources to recruit onlookers. The food sources found by all employed bees are selected and exploited by onlookers according to the probability proportional to the quality of food sources. Scouts are generated from a few employed bees, which abandon their food sources through a predetermined number of cycles called limit and search new ones. The position of each food source is a possible solution to an optimization problem, and its fitness is the profitability of that food source. The number of food sources equals to the number of employed bees. Initially, a population containing $\mathrm{SN}$ solutions is generated randomly. SN is the number of food sources, which is half of the population size (NP). Let $X_{i}=x_{i 1}, x_{i 2}, \ldots, x_{i D}$ represents the $i$ th food source in the population, where $D$ is the number of optimization parameters. The population is subject to repeated cycles, $C=1,2, \ldots$, maximum cycle number $(\mathrm{MCN})$, of the search processes of the employed bees, onlookers and scouts. in $\mathrm{ABC}$, the fitness function is defined as $\operatorname{Eq}(5)$

$$
\text { fit }\left(X_{i}\right)=\left\{\begin{array}{cl}
\frac{1}{1+f\left(X_{i}\right.} & \text { if } f\left(X_{i}\right) \geq 0 \\
1+a b s\left(f\left(X_{i}\right)\right) & \text { if } f\left(X_{i}\right)<0
\end{array}\right.
$$

where $f\left(X_{i}\right)$ is the value of objective function of $X_{i}, f i t\left(X_{i}\right)$ is the fitness value of $X_{i}$. The probability of a food source being selected by an onlooker can be represented by $\operatorname{Eq}(6)$

$$
p\left(X_{i}\right)=\frac{f i t\left(X_{i}\right)}{\sum_{n=1}^{S N} f i t\left(X_{n}\right)}
$$

Afetr discovering or selecting the food source $X_{i}$ by an employed bee or an onlooker, they exploit a neighboring food source $V_{i} . V_{i}$ is determined by changing only one parameter of $X_{i}$, where $v_{i j} \neq x_{i j}$, while the rest of $V_{i}$ keep the same value as $X_{i}, v_{i j}$ is generated by Eq (7)

$$
v_{i j}=x_{i j}+\phi_{i j}\left(x_{i j}-x_{k j}\right)
$$

where $k \in 1,2, \ldots, \mathrm{SN}$ is a random chosen inde and $k$ must be different from from $i, j \in 1,2, \ldots, \mathrm{D}, \phi_{i j}$ is a random number between $[-1,1]$. After determining a new candidate food source in the neighborhood of its currently associated food source using Eq (7) by an employed bee or onlooker, a greedy selection method is used to distinguish between the new food source and the old one. if the abandoned food source is $X_{i}$, a scout produces a new food source according to Eq (8)

$$
v_{i j}=x_{m i n j}+\operatorname{rand}(0,1)\left(x_{\max j}-x_{\text {minj }}\right)
$$

where $x_{\operatorname{minj}}$ and $x_{\max j}$ are the lower and upper bounds of the variable $x_{i j}$, respectively.

$\mathrm{ABC}$ is a possible technique to optimize the parameters of ANFIS. In ANFIS-ABC, ANFIS parameters are considered as one food source that represent a possible solution, and parameters that affect the ANFIS training can be taken as the dimensions of each food source.

Two parameters in ANFIS are to be optimized which are the linguistic hedges $p$ that affect the membership function values and the consequent parameters $k$ that accilerates the performances value.

Denote $X$ as a food source, and let $p$ denotes the set of linguistic hedges of each input where $p \in$ $\{$ verylow, low, medium,...$\}$. Each linguistic hedge $p$ is presented by gaussian membership function $\mu^{\text {gaussian }}$ whose parameters are center $m$ and the width $\sigma$ as shown in Eq (1). The number of linguistic hedges per input equals the number of ANFIS rules $R$. Then an algebraic representation of the antecedent parameters is shown in Eq (9).

$$
X=\left\{\mu_{j r}^{\text {gaussian }} \mid r \in R ; j \in J\right\}
$$

where $J$ is a set of inputs and $R$ is a set of rules that forms ANFIS-ABC.

Let $k$ be denoted as the consequent parameters of each food source. The consequent parameters $k$ of each rule output presents the parameters of a linear membership function $\mu^{\text {linear }}$ whose the number of parameters equals $\mathrm{J}+1$. Then an algebraic representation of the length of the dimension formed by $k$ is shown in $\operatorname{Eq}(10)$.

$$
X=\left\{\mu_{r}^{\text {linear }} \mid r \in R\right\}
$$


Because the dimensions of one food source in the ANFIS$\mathrm{ABC}$ has two parameters, then an algebraic representation of that one food source is presented in $\mathrm{Eq}(11)$.

$$
X=\left\{\left(\mu_{j r}^{\text {gaussian }}, \mu_{r}^{\text {linear }}\right) \mid j \in J ; r \in R\right\}
$$

Fig (2) shows The coding of antecedent and consequent parameters in each food source. The same as ABC, the ANFIS-

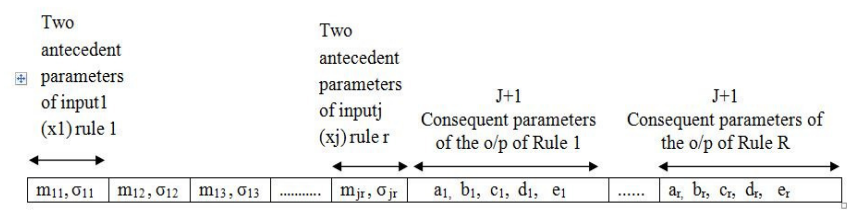

Fig. 2: The coding of ANFIS-ABC source

ABC consists of a number of food sources. Then an algebraic representation of the dimension of each food source in the ANFIS-ABC is represented in Eq (12).

$$
X_{i}=\left\{\left(\mu_{i j r}^{\text {gaussian }}, \mu_{i r}^{\text {linear }}\right) \mid j \in J ; r \in R\right\}
$$

where $i=1,2, \ldots \mathrm{N}$ and $\mathrm{N}$ is the number of food sources. ANFIS$\mathrm{ABC}$ requires an objective function to minimize which is the root mean square error (RMSE) of ANFIS structure. Eq(13) shows the RMSE calculation.

$$
R M S E=\sqrt{\frac{\sum_{i=1}^{s}\left(\hat{y}_{i}-y_{i}\right)^{2}}{s}}
$$

where $y_{i}$ is the observed value for the ith observation and $\hat{y}_{i}$ is the predicted output from fuzzy model, and $\mathrm{s}$ is the number of training data pairs. Fig (3) shows the whole ANFISABC model. Fig (4) shows the antecedent part of ANFIS-ABC

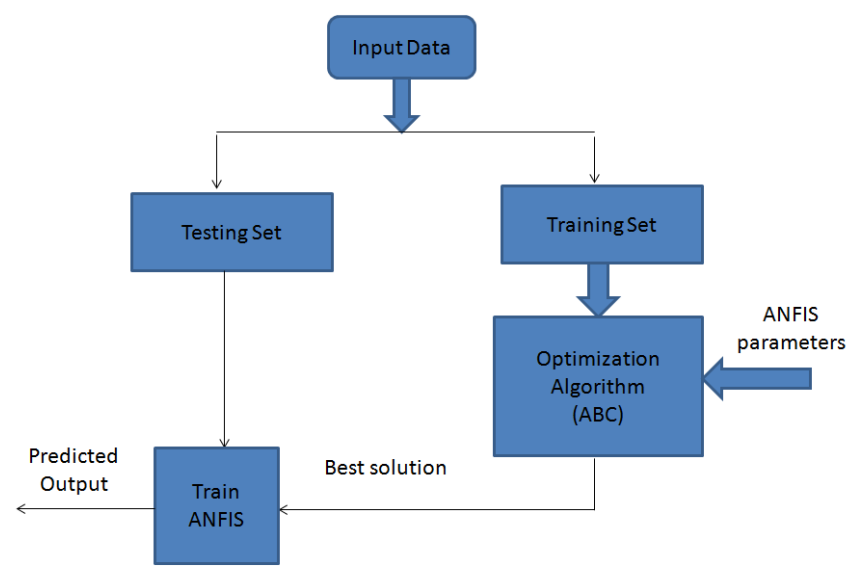

Fig. 3: ANFIS-ABC Structure

parameters and the process of generating a firing strength for each output rule. Fig (5) shows the consequent part of ANFIS$\mathrm{ABC}$ and generating the final crisp output. Fig (6) shows how the objective function (RMSE) of the ANFIS-ABC model is calculated and then converted into fitness value. Fig (7) shows

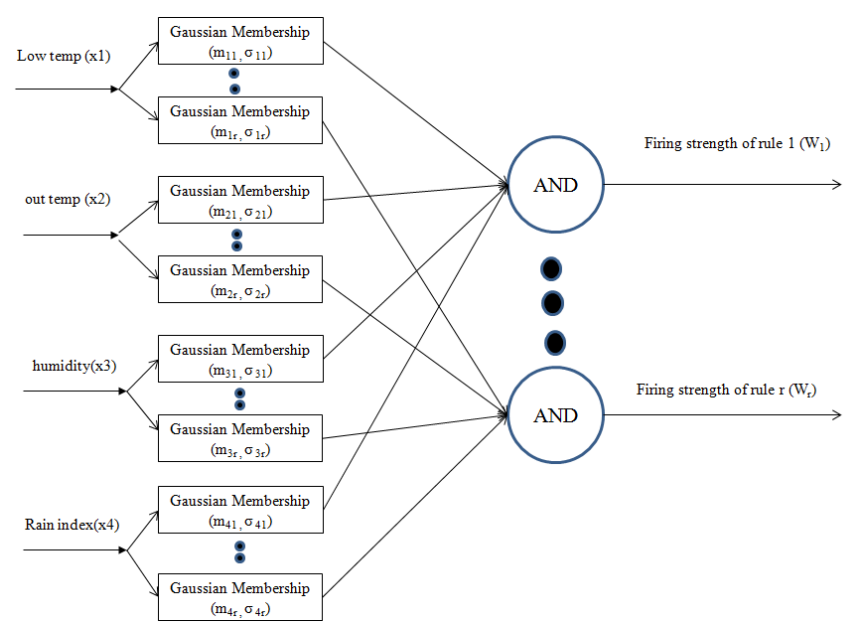

Fig. 4: Antecedent Part of ANFIS-ABC

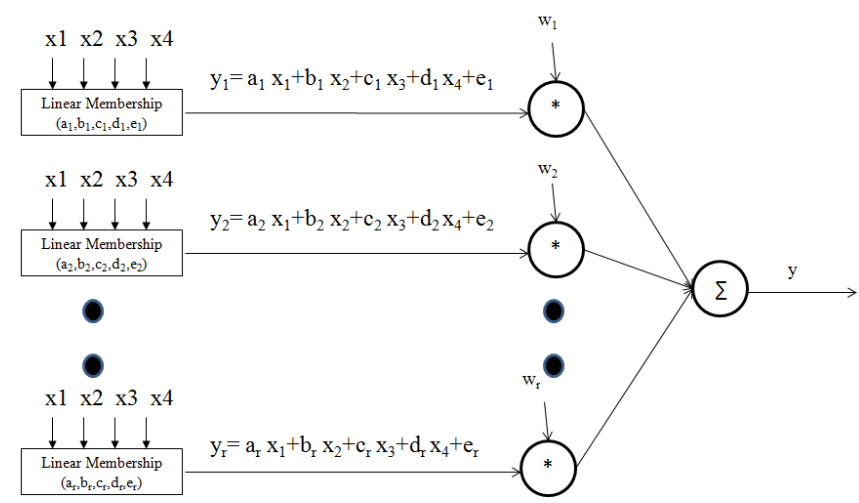

Fig. 5: Consequent Part of ANFIS-ABC

the proposed ANFIS-ABC structure and The main steps of ANFIS-ABC are outlines below as in algorithm 1:

\section{EXPERIMENTAL RESULTS}

Seventy percent of the data set is used in training ANFISABC model. The parameters initialization and the achieved results are discussed in this section.

\section{A. Data set description}

Each instance of the data set consists of four inputs and only one output. The four inputs are the low temperature, the out temperature, the humidity and the rain index and are represented as $(\operatorname{In} 1, \operatorname{In} 2, \operatorname{In} 3$ and $\operatorname{In} 4)$. The output value indicates the wind speed. Only $70 \%$ of the dataset is used in training the model which constitutes 2128 records out of 3040 records.

\section{B. ANFIS parameters}

The ANFIS model parameters are shown in table I. The ANFIS-ABC model was trained four times with different number of rules. In this training procedure, the ANFIS-ABC algorithm is used to optimise both the antecedent parameters 


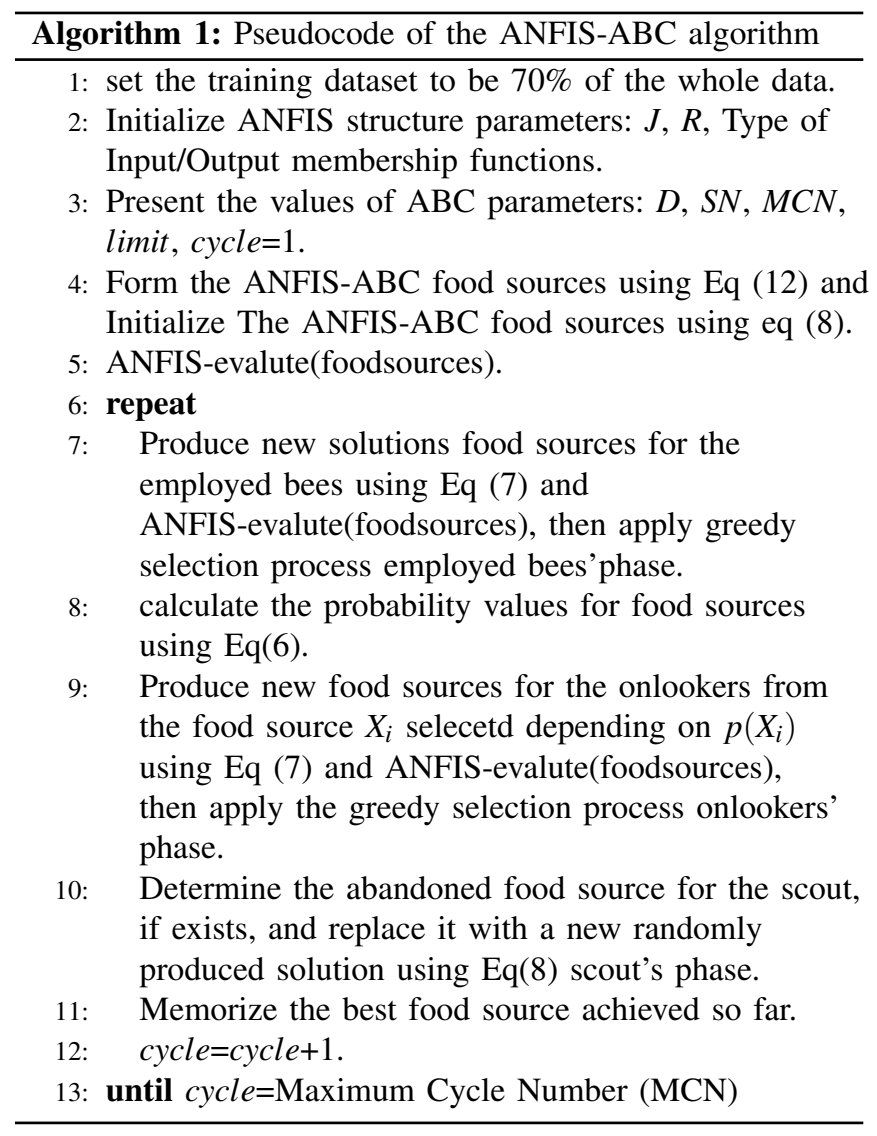

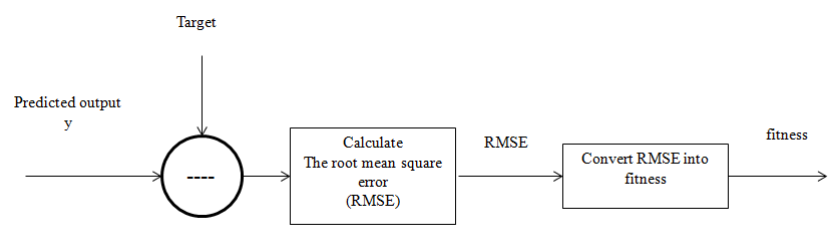

Fig. 6: Evaluating the output of ANFIS-ABC

TABLE II: ABC Parameters

\begin{tabular}{|l|c|}
\hline \multicolumn{2}{|c|}{ ABC Parameters } \\
\hline Number of Optimization Parameters (D) & $\mathrm{J} * \mathrm{R} * 2+\mathrm{R}(\mathrm{J}+1)$ \\
\hline Number of Food Sources (SN) & 120 \\
\hline Number of Employed Bees (Ebees) & 60 \\
\hline Number of Onlookers (Onbees) & 60 \\
\hline limit (L) & round $\left(0.6^{*} \mathrm{D}^{*}\right.$ Ebees) \\
\hline Maximum Cycle Number (MCN) & 300 \\
\hline
\end{tabular}

and the consequent parameters. A Gaussian membership function is used in the input layer and linear membership function is used in the output layer. The number of variables in each food source will depends on the number of parameters of membership function for each input and output. The consequent part of each $z_{i}$ Suegno ANFIS rule will take the form $k_{1} * \operatorname{In} 1+k_{2} * \operatorname{In} 2+k_{3} * \operatorname{In} 3+k_{4} * \operatorname{In} 4+k_{5}$. where $k_{1}, k_{2}, k_{3}, k_{4}$ and $k_{5}$ are the consequent parameters of each rule output.

\section{ABC parameters}

the $\mathrm{ABC}$ parameters are initialized as shown in table II

D. Training ANFIS-ABC system with different number of membership functions

The system has been trained using different number of rules, hence different number of membership functions per input/output. The objective of the training is to obtain the parameters of membership functions that achieve least mean square error value. The results are shown in table III. It is worth to note that the increase in the number of membership functions does not improve the value of RMSE. The case of using four membership functions shows a slight improvement.

\section{Wind SPEEd PREDiction System}

TABLE I: ANFIS Parameters

\begin{tabular}{|l|c|}
\hline \multicolumn{2}{|c|}{ ANFIS Parameters } \\
\hline Number of Crisp Inputs (J) & 4 \\
\hline Input Membership Functions Type & Gaussian \\
\hline $\begin{array}{l}\text { Number of optimized Parameters } \\
\text { of Gaussian Membership Function }\end{array}$ & 2 \\
\hline Output Membership Function Type & Linear \\
\hline $\begin{array}{l}\text { Number of Optimized Parameters } \\
\text { of Linear Membership Function }\end{array}$ & J+1 \\
\hline Number of Output Rules (R) & 3 or 4 or 5 or 10 \\
\hline Number of Fuzzy Sets per Input & 3 or 4 or 5 or 10 \\
\hline
\end{tabular}

The optimized ANFIS parameters obtained by ABC are used to build and test a system for wind speed prediction. Thirty percentage of the data is used for testing the prediction

TABLE III: Results of Training ANFIS-ABC model

\begin{tabular}{|c|c|}
\hline \multicolumn{2}{|c|}{ Training ANFIS-ABC Model } \\
\hline Number of Membership Functions & RMSE \\
\hline 3 & 0.530 \\
\hline 4 & 0.440 \\
\hline 5 & 0.410 \\
\hline 10 & 0.390 \\
\hline
\end{tabular}




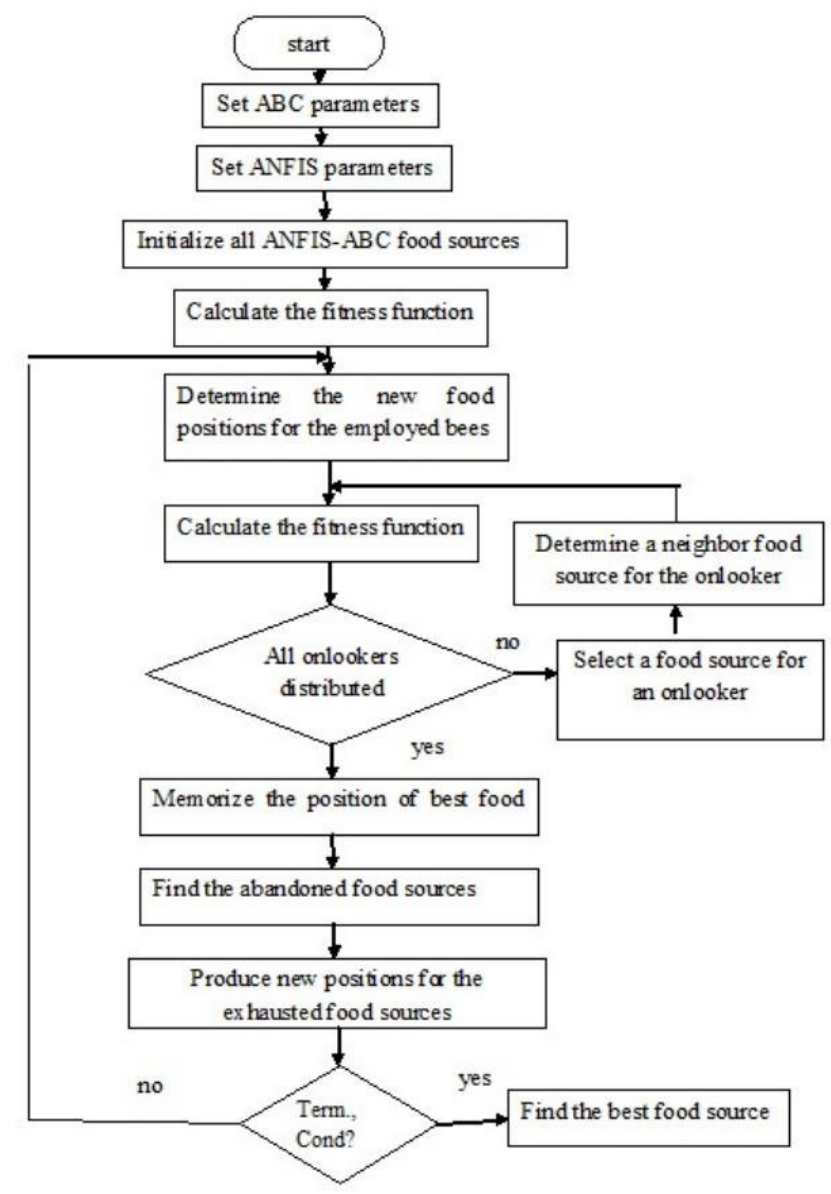

Fig. 7: Proposed ANFIS-ABC structure

system. Different number of membership functions are used along with 4 input parameters (low temeperature, out temperature, humidity and rain index). The accuracy of the resulting system is discussed below.

\section{A. Wind Speed Prediction with 3 Membership Functions}

Three membership functions means three linguistic variables low,medium and high for the measurements of the values of each input parameter. Fig 8 shows the ANFIS(ABC) structure and Fig 9 shows the generated system which can be used to predict the wind speed (output) by enter four input values corresponding to the input parameters. The average testing error is 0.29 which indicates accuracy with $71 \%$. Fig $(4,5)$ show the ANFIS-ABC structure and output with three rules.

\section{B. Wind Speed Prediction with 4 Membership Functions}

Four membership functions means four linguistic variables very low, low, medium and high for the measurements of the values of each input parameter. Fig 10 shows the ANFIS(ABC) structure and Fig 11 shows the generated system which can be used to predict the wind speed (output) by enter four input

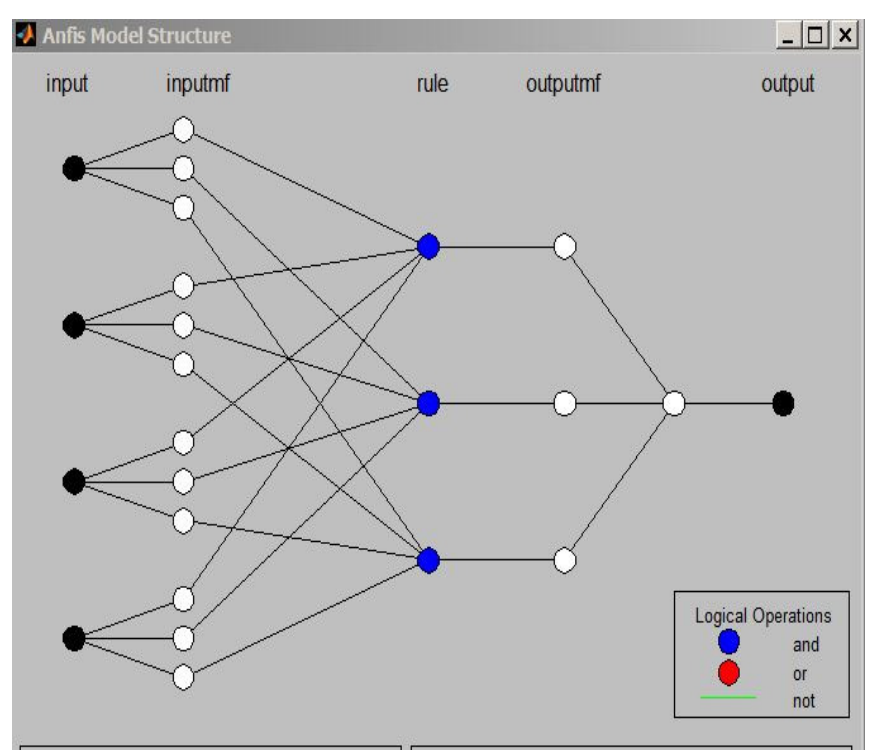

Fig. 8: ANFIS-ABC Structure with three rules

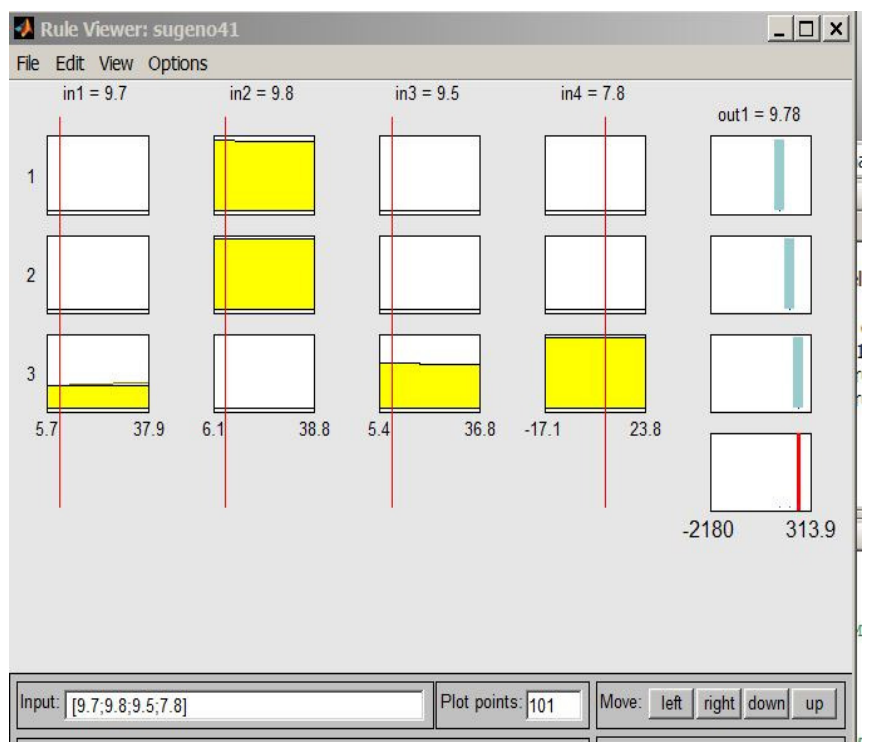

Fig. 9: Wind Prediction with three rules

values corresponding to the input parameters. The average testing error is 0.30 which indicates accuracy with $70 \%$.

\section{Wind Speed Prediction with 5 Membership Functions}

Five membership functions for each input means five linguistic variables very low, low, medium, high and very high for the measurements of the values of each input parameter. Fig 11 shows the ANFIS(ABC) structure and Fig 12 shows the generated system which can be used to predict the wind speed (output) by enter five input values corresponding to the input parameters. The average testing error is 0.29 which indicates accuracy with $71 \%$. 


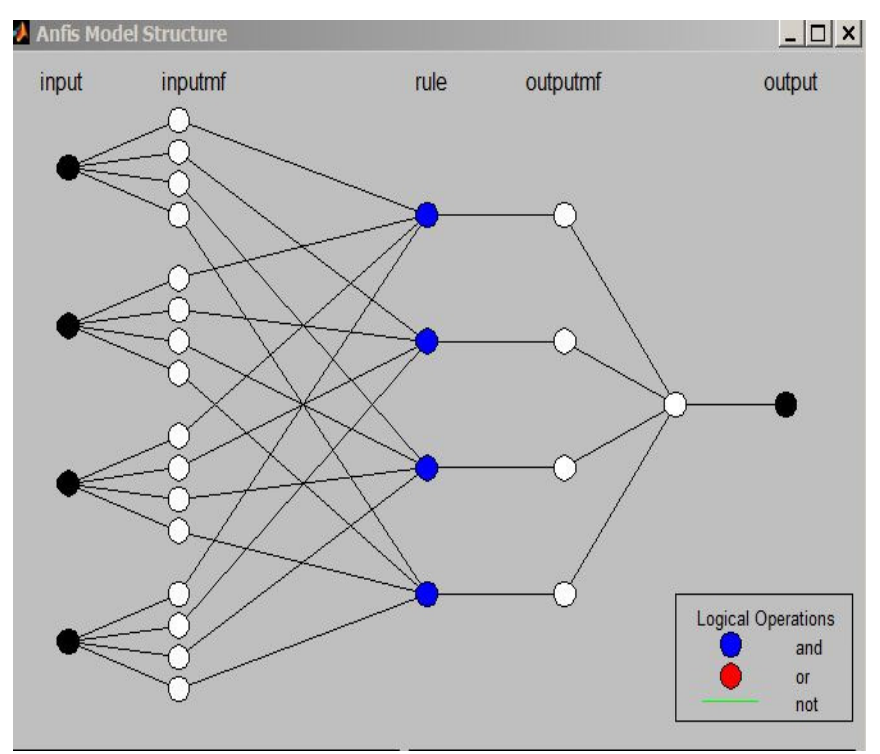

Fig. 10: ANFIS-ABC Structure with four rules

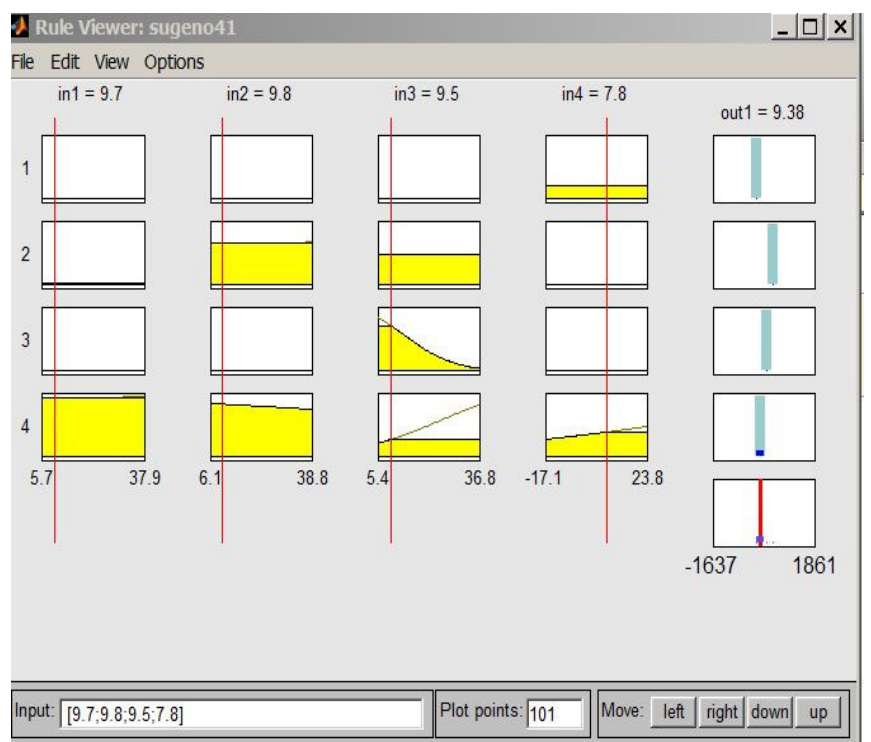

Fig. 11: Wind Prediction with four rules

\section{Wind Speed Prediction with 10 Membership Functions}

Ten membership functions for each input means Ten different classes for the measurements of the values of each input parameter. Fig 14 shows the generated system which can be used to predict the wind speed (output) by enter four input values corresponding to the input parameters. The average testing error is 0.29 which indicates accuracy with $71 \%$. from the above discussion, It is deduced that the best achieved accuracy is $70 \%$ with four membership functions. The increase of membership functions does not improve the accuracy significantly. Table IV shows the summary of achieved results.

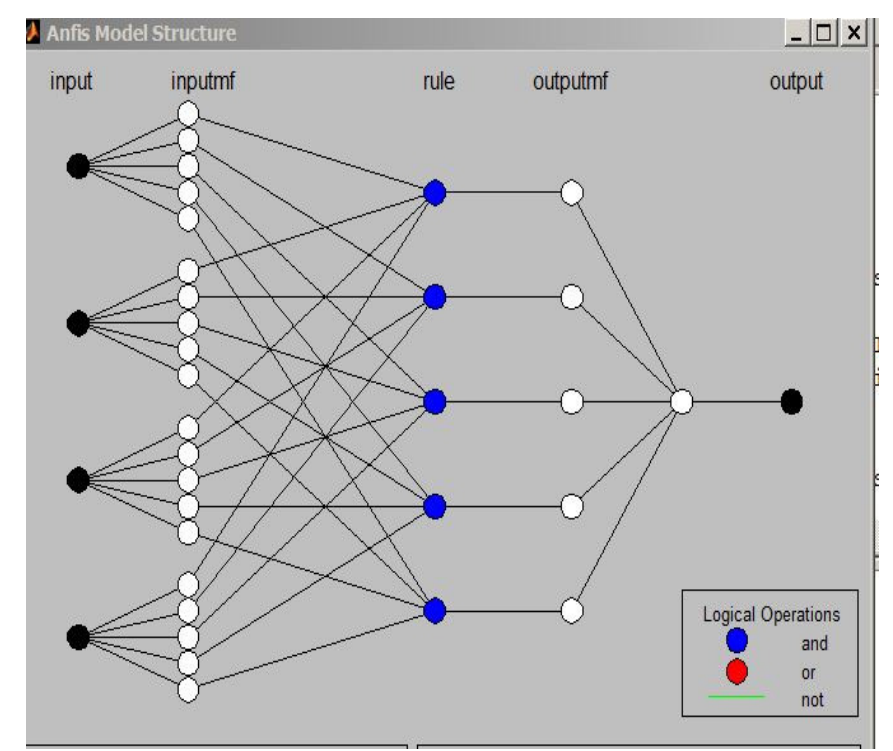

Fig. 12: ANFIS-ABC Structure with five rules

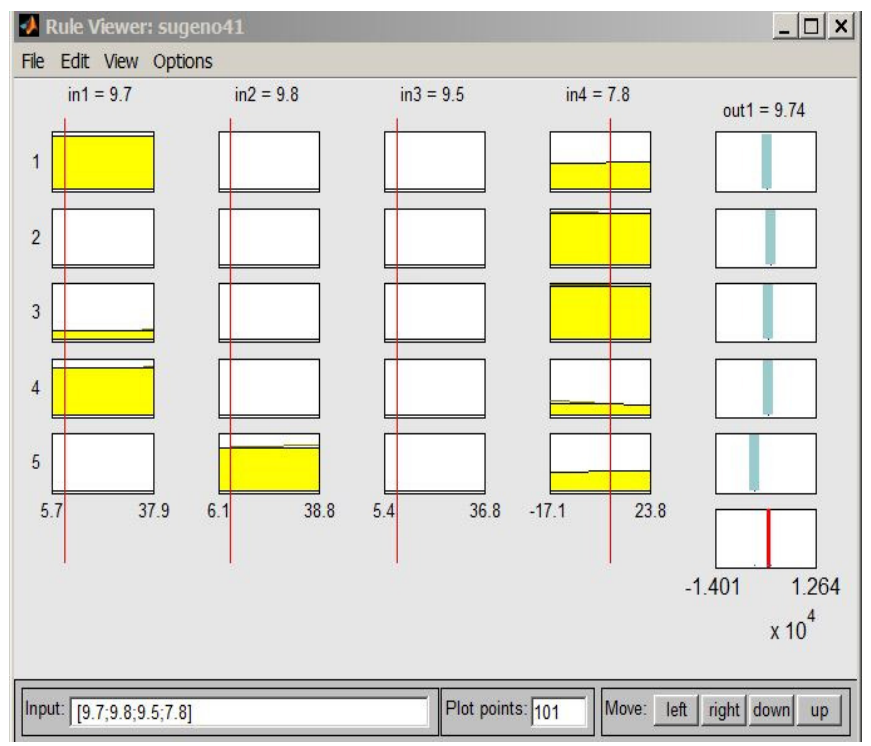

Fig. 13: Wind Prediction with five rules

\section{CONCLUSION AND FUTURE WORKS}

A new approach for optimising the Sugeno adaptive neurofuzzy inference system (ANFIS) in prediction problems has been proposed in this paper. The ABC technique is integrated into the process of ANFIS in order to achieve the optimal solution for ANFIS. This was achieved by simultaneously optimising the ANFIS performance based on a criteria which is enhancing the accuracy based on lower error rate. The experimental results indicated that ANFIS-ABC provides a promised accuracy in prediction problems. However, an algorithm that can result in a complete balance of accuracy and interpretability would be more adaptable for real applications. 
TABLE IV: Results of Testing ANFIS-ABC

\begin{tabular}{|c|c|c|c|}
\hline \multicolumn{4}{|c|}{ Results of Testing ANFIS-ABC } \\
\hline $\begin{array}{c}\text { Num of Membership } \\
\text { Functions }\end{array}$ & MAE & MAPE\% & Accuracy\% \\
\hline 3 & 0.43 & $2.6 \%$ & $97.4 \%$ \\
\hline 4 & 0.33 & $2.4 \%$ & $97.6 \%$ \\
\hline 5 & 0.3 & $1.9 \%$ & $98.1 \%$ \\
\hline 10 & 0.29 & $1.8 \%$ & $98.2 \%$ \\
\hline
\end{tabular}

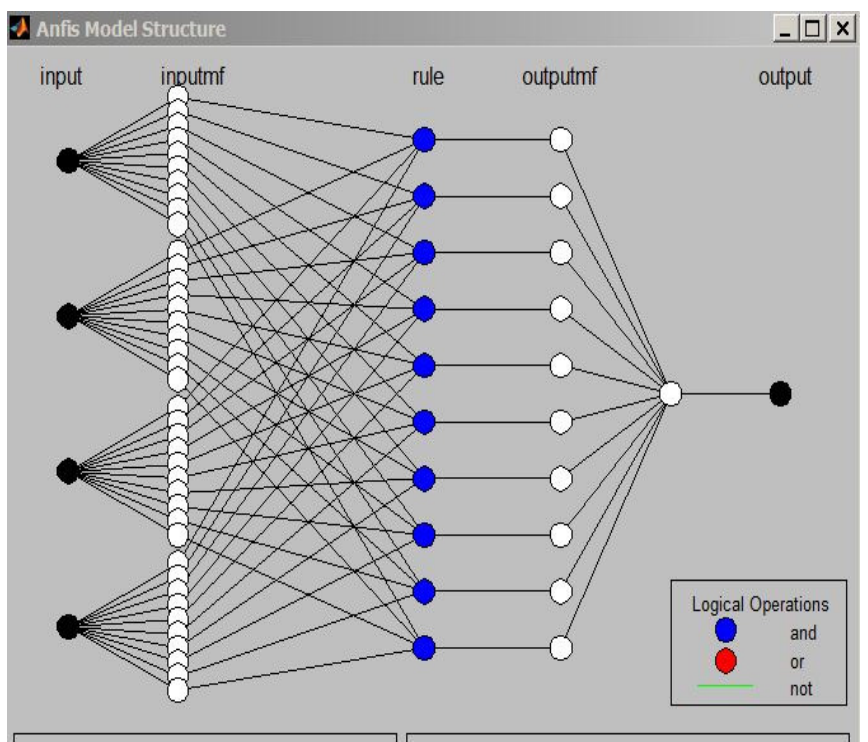

Fig. 14: ANFIS-ABC Structure with ten rules

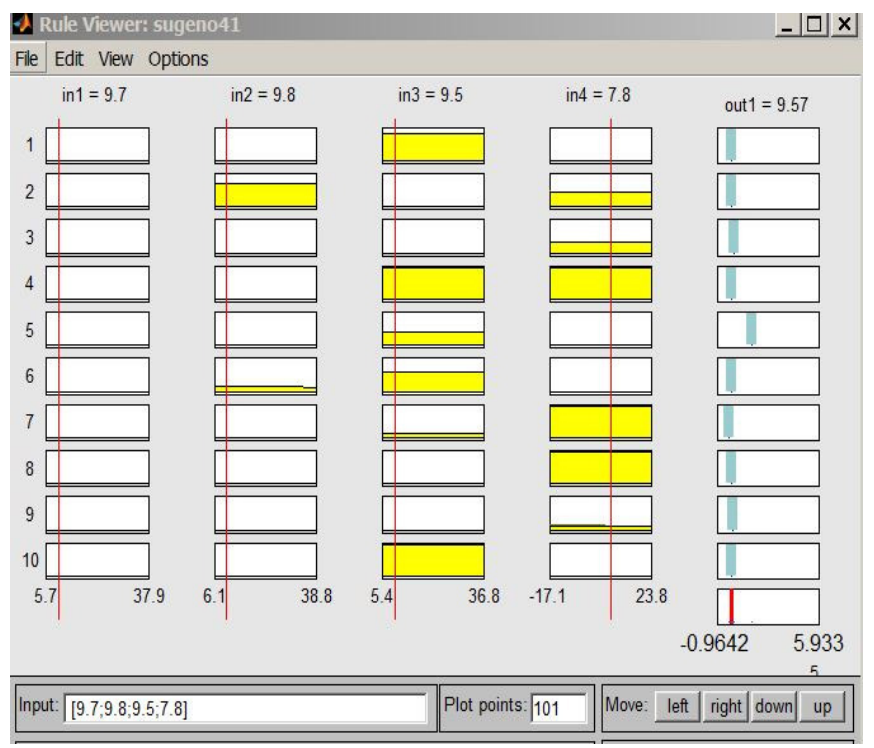

Fig. 15: Wind Prediction with ten rules
Thus, problems based on this approach are the subject of further work by integrating other swarm algorithms.

\section{REFERENCES}

[1] Jang J. S. R. Adaptive network based fuzzy inference systems. IEEE Transactions on systems man and cybernetics 1993, p. 665-685.

[2] Capovilla CE, Casella IRS, Filho AJS, Azcue-Puma JL, Jacomini RV, Ruppert E. A wind energy generator for smart grid applications using wireless-coded neuro-fuzzy power control. Comput Math Appl 2014;68:2112-23.

[3] De Giorgi MG, Ficarella A, Tarantino M. Error analysis of short term wind power prediction models. Appl Energy 2011;88:1298-311.

[4] Haque AU, Mandal P, Kaye ME, Meng J, Chang J, Senjyu T. A new strategy for predicting short-term wind speed using soft computing models. Renew Sustain Energy Rev 2012;16:4563-73.

[5] Petković D, Zarko C, Nikolic V. Adaptive neuro-fuzzy approach for wind turbine power coefficient estimation. Renew Sustain Energy Rev 2013;28:191-5.

[6] Jafarian M, Ranjbar AM. Fuzzy modeling techniques and artificial neural networks to estimate annual energy output of a wind turbine. Renew Energy 2010;35:2008-14.

[7] Lin WM, Hong CM, Cheng FS. Fuzzy neural network output maximization control for sensorless wind energy conversion system. Energy 2010;35:592-601.

[8] Lin WM, Hong CM, Cheng FS. Design of intelligent controllers for wind generation system with sensorless maximum wind energy control. Energy Convers Manag 2011:52:1086-96.

[9] Mohandes M, Rehman S, Rahman SM. Estimation of wind speed profile using adaptive neuro-fuzzy inference system (ANFIS). Appl Energy 2011;88:4024-32.

[10] De Giorgi MG, Ficarella A, Tarantino M. Error analysis of short term wind power prediction models. Appl Energy 2011;88:1298-311.

[11] Monfared M, Rastegar H, Kojabadi HM. A new strategy for win speed forecasting using artificial intelligent methods. Renew Energy 2009;34:845-8.

[12] Karaboga, D.; Akay, B. A survey: algorithms simulating bee swarm intelligence. Artif. Intell. Rev. 2009, 31, 61-85.

[13] D. Karaboga, B. Basturk, A powerful and efficient algorithm for numerical function optimization: artificial bee colony (ABC) algorithm, Journal of Global Optimization 39(2007) 171-459.

[14] Karaboga, D.; Basturk, B. On the performance of artificial bee colony (ABC) algorithm. Appl. Soft Comput. 2008, 8, 687-697.

[15] Karaboga, D.; Akay, B. A comparative study of artificial bee colony algorithm. Appl. Math. Comput. 2009, 214, 108-132.

[16] Karaboga, D.; Basturk, B. A powerful and efficient algorithm for numerical function optimization: artificial bee colony $(\mathrm{ABC})$ algorithm. J. Glob. Optim. 2007, 39, 459-471.

[17] Kang, F.; Li, J.; Xu, Q. Structural inverse analysis by hybrid simplex artificial bee colony algorithms. Comput. Struct. 2009, 87, 861-870.

[18] Sonmez, M. Discrete optimum design of truss structures using artificial bee colony algorithm. Struct. Multidiscip. Optim. 2011, 43, 85-97.

[19] Samanta, S.; Chakraborty, S. Parametric optimization of some nontraditional machining processes using artificial bee colony algorithm. Eng. Appl. Artif. Intell. 2011, 24, 946-957. 